\title{
EFECTOS DE LA SUPLEMENTACIÓN ORAL YEJERCICIOS DE RESISTENCIA DURANTE LA SESIÓN DE HEMODIÁLISIS EN PACIENTES DEL HOSPITAL INFANTIL DR. ROBERT REID CABRAL, PERÍODO ABRIL-JUNIO 2019 \\ Effects of oral supplementation and resistance exercises during the hemodialysis session in patients of the Children's Hospital Dr. Robert Reid Cabral, April-June 2019
}

\section{Willna De la Rosa Brito ${ }^{a}$ Ankia Mora ${ }^{b}$, Manuel Colomé-Hidalgo ${ }^{c}$ Y Yun Zyong Kim ${ }^{d}$}

Recibido: 12 de diciembre, $2020 \bullet$ Aprobado: 12 de marzo, 2021

Cómo citar: De la Rosa Brito W, Mora A, Colomé Hidalgo M, Zyong Kim Y. Efectos de la suplementación oral y ejercicios de resistencia durante la sesión de hemodiálisis en pacientes del Hospital Infantil Dr. Robert Reid Cabral, período abril-junio 2019. cysa [Internet]. [citado 22 de febrero de 2022];6(1):71-9. Disponible en: https://revistas.intec.edu.do/index.php/cisa/article/view/2403

\section{Resumen}

Se realizó un estudio tipo descriptivo, prospectivo, de corte transversal, con el objetivo de establecer los efectos de la suplementación oral y ejercicios de resistencia en pacientes de hemodiálisis Hospital Infantil Dr. Robert Reid Cabral abril-junio 2019. La población estuvo conformada por 60 pacientes con Enfermedad Renal Crónica perteneciente al servicio de diálisis, mientras que la muestra estuvo constituida por 17 pacientes. Finalizamos el proyecto con 11 pacientes, en vista de que en el periodo descrito se trasladaron 3 a diálisis peritoneal, 1 fue referido a adulto, 1 fallecido, 1 abandonó el estudio. La técnica utilizada para el levantamiento de la información fue un cuestionario de recolección de datos, procesados con el programa estadístico SPSS, utilizándose la prueba de Wilcoxon, donde el contraste de hipótesis es comparado con el valor $\mathrm{p}=0.05$, un nivel de confianza del $95 \%$.

Los hallazgos más relevantes mostraron que la edad más frecuente fue 17 años, El sexo más frecuente el masculino;

\footnotetext{
${ }^{a}$ Nefróloga Pediatra, Hospital Infantil Dr. Robert Reid Cabral, Santo Domingo, República Dominicana. ORCID: 0000-0001-6470-5112, Correo-e: willnadelarosa@gmail.com

${ }^{\mathrm{b}}$ Nefróloga Pediatra, Hospital Infantil Dr. Robert Reid Cabral.

ORCID: 0000-0003-1264-8181, Correo-e: amora@macrotech.com.do
}

\begin{abstract}
A descriptive, prospective, cross-sectional study was carried out to establish the effects of oral supplementation and resistance exercises in hemodialysis patients Hospital Infantil Dr. Robert Reid Cabral April-June 2019. The population consisted of 60 patients with Chronic Kidney Disease belonging to the dialysis service, while the sample consisted of 17 patients. We finished the project with 11 patients, given that in the period described, 3 were transferred to peritoneal dialysis, 1 was referred to an adult dialysis program, 1 died, and 1 dropped out of the study. The technique used to collect the information was a data collection questionnaire, processed with the statistical program SPSS, using the Wilcoxon test, where the contrast of hypotheses is compared with the value $\mathrm{p}=0.05$, a confidence level of $95 \%$.

The most relevant findings showed that the most frequent age was 17 years, the most frequent sex was male; an average

\footnotetext{
c Gerente del Servicio de Epidemiología, Hospital Pediátrico Dr. Hugo Mendoza, República Dominicana. ORCID: 0000-0002-4562-6491,

${ }^{\mathrm{d}}$ Gerente de Servicio de Nutrición Hospital Infantil Dr. Robert Reid Cabral y Coordinadora de Maestría en Nutriología Clínica Pediátrica en Instituto Tecnológico de Santo Domingo, República Dominicana. ORCID: 0000-0001-9381-2797, Correo-e: kim@intec.edu.do
} Correo-e: manuel.colome@hhm.gob.do
\end{abstract}


un promedio del $63.6 \%$ tomaron del $90-100 \%$ del suplemento indicado. El promedio de ejercicios realizado fue de 20-30 minutos. Los signos y síntomas frecuentes como efecto secundario a la realización de ejercicios se presentaron en 3 pacientes: dolor muscular; y vómito y dolor abdominal para la ingesta del suplemento. El peso tuvo un rango promedio de 3-5 \% ganado. Para el índice de masa corporal el porcentaje ganado fue 1-6 \%. La evolución de la masa muscular tuvo un promedio ganado de $11-15 \%$ En la evolución de la masa grasa $36 \%$ perdieron entre 6-14 \% de grasa. Tanto la hemoglobina como el hemato6-14 \% de grasa. Tanto la hemoglobina como el hematocrito aumentaron en el $100 \%$ de los pacientes. Los niveles de urea, proteínas y albúmina no evidenciaron diferencias significativas. En conclusion, demostramos en este estudio que administrando suplementación oral intradiacosión corporal de los pacientes en hemodiálisis.

Palabras clave: suplementación oral, ejercicios de resistencia, hemodiálisis, pediatría

\section{Introducción}

La función renal juega un papel en la regulación del equilibrio ácido-base, balance hidroeléctrico, metabolismo fosfocálcico y balance nitrogenado. Por ello, la enfermedad renal aguda o la enfermedad renal crónica (ERC) afectan de una manera especial la situación metabólica nutricional de los pacientes. Los nińos con ERC presentan una alta prevalencia de malnutrición calórico-proteica, con alteración del compartimiento graso y proteico, así como una profunda alteración de las proteínas séricas. ${ }^{1}$

Se ha estimado que existe malnutrición energéticoproteica (MEP) en aproximadamente el $20-50 \%$ de los pacientes de diálisis, que puede deberse a diferentes causas, incluyendo la ingesta insuficiente de nutrientes en la dieta, el hipercatabolismo propio de la diálisis, la acidosis metabólica, la inflamación crónica y las alteraciones hormonales. La mayoría de los pacientes con ERC presentan el llamado síndrome de malnutrición, inflamación y anemia (MIA), que incluye una baja concentración de proteínas séricas y pérdida de masa magra, asociado of $63.6 \%$ took $90-100 \%$ of the indicated supplement. The average number of exercises performed was 20-30 minutes. Frequent signs and symptoms as a side effect of exercising were presented muscle pain in 3 patients. In relation to oral supplements were vomiting and abdominal pain. The weight had an average range of 3-5\% gained and for the body mass index, the percentage gained was $1-6 \%$. The evolution of muscle mass had an average gain of $11-15 \%$. In the evolution of fat mass, $36 \%$ lost between $6-14 \%$ of fat. Both hemoglobin and hematocrit increased in 100\% of patients. Urea, protein, and albumin levels did not show significant differences. In conclusion, we demonstrate in this study that adminitering in tion, together wh emodialysis patients.

Keywords: oral supplementation, resistance exercises, hemodialysis, pediatric

a un aumento de las concentraciones de los marcadores inflamatorios. ${ }^{2}$

Los estudios recientes indican que la suplementación de nutrientes, por vía oral o parenteral durante el proceso de diálisis, podrían compensar la ingesta relativamente inadecuada de proteínas y de energía. En las líneas guía de la European Society for Parenteral and Enteral Nutrition (ESPEN) se establece que los suplementos orales son la vía preferente de realimentación para los pacientes en hemodiálisis. ${ }^{3}$

Como consecuencia de la enfermedad renal crónica, se ven afectados el sistema cardiovascular y músculo esquelético, que consecuentemente disminuyen la capacidad funcional del paciente. Desde 1980 es conocida la importancia del ejercicio físico en pacientes en hemodiálisis, siendo una de las modalidades intradiálisis. Bernard y Holloszy refieren el incremento del número de mitocondrias como efecto de la práctica sistemática de actividades físicas, que se traduce en mayores posibilidades para consumir oxígeno. ${ }^{4}$

\section{Material y métodos}

El estudio se realizó en la unidad de hemodiálisis del Hospital Infantil Dr. Robert Reid Cabral, en la ciudad de Santo Domingo, República Dominicana.

La población estuvo conformada por 60 pacientes con Enfermedad Renal Crónica perteneciente al servicio de diálisis, en el periodo abril-junio 2019. De los cuales, 17 pacientes, de ambos sexos, están registrados en el programa de hemodiálisis. Completamos el estudio con 11 pacientes, en vista de que en el periodo descrito se trasladaron 3 pacientes a diálisis peritoneal, 1 fue referido a adulto, 1 fallecido, y 1 abandonó el estudio. Estos pacientes constituyen nuestro objeto de estudio y en ellos evaluamos las siguientes variables: edad, sexo, suplementación oral, ejercicios de resistencia, peso, Índice de masa corporal, masa muscular y grasa, síntomas (tolerancia del suplemento y los ejercicios), pruebas de laboratorio.

Los datos fueron organizados y tabulados mediante el paquete estadístico SPSS, procediendo a la elaboración de cuadros y gráficos. Para tales fines se utilizó el programa Microsoft Office (Excel). Para el contraste de hipótesis no paramétrico de pruebas pareadas para distribuciones libres se utilizó el estadístico de signos de Wilcoxon.

\section{Resultados}

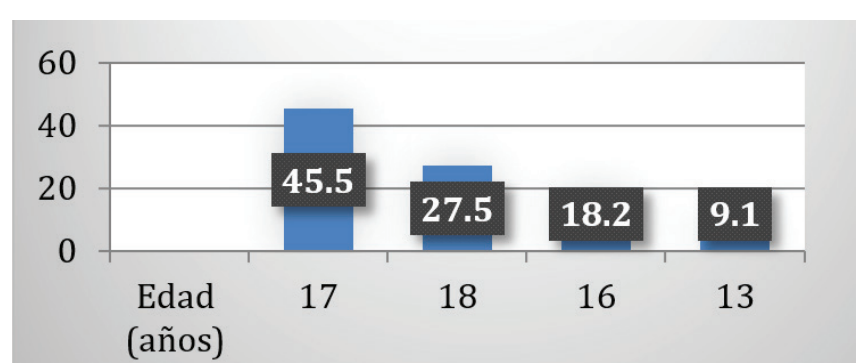

Figura 1. Edad de los pacientes en la unidad de hemodiálisis del Hospital Infantil Dr. Robert Reid Cabral, periodo abril-junio 2019

Fuente: elaboración propia.

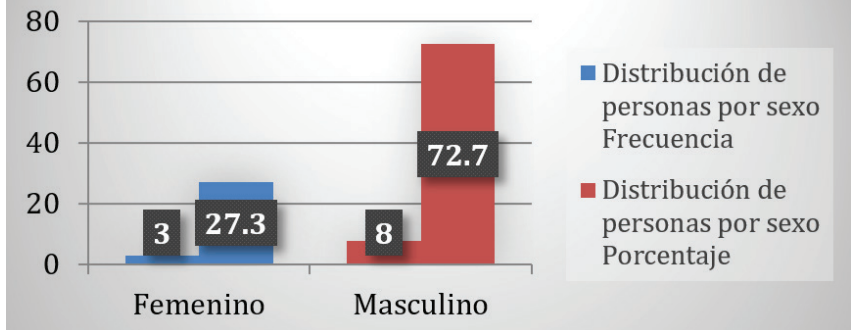

Figura 2. Sexo de los pacientes en la unidad de hemodiálisis del Hospital Infantil Dr. Robert Reid Cabral en el periodo abril-junio 2019

Fuente: elaboración propia.
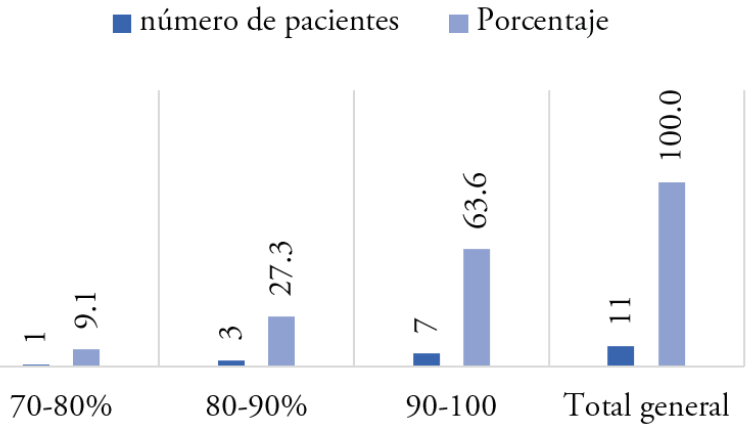

Figura 3. Promedio de suplemento tomado durante la sesión de hemodiálisis en pacientes del Hospital Infantil Dr. Robert Reid Cabral, periodo abril-junio 2019

Fuente: elaboración propia.

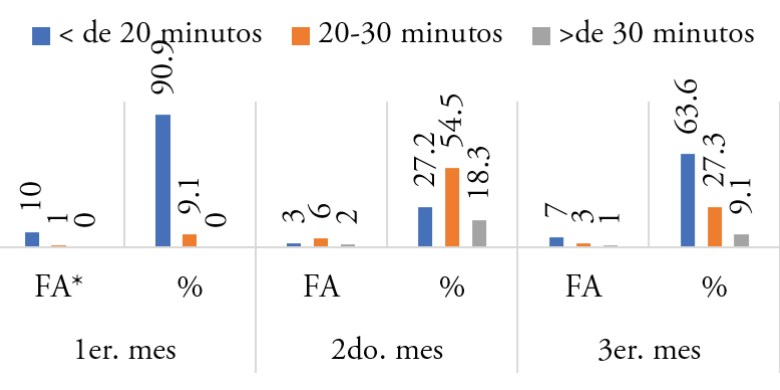

Figura 4. Promedio del ejercicio de resistencia realizado por mes en los pacientes de la unidad de hemodiálisis del Hospital Infantil Dr. Robert Reid Cabral, periodo abril-junio 2019

Fuente: elaboración propia. 


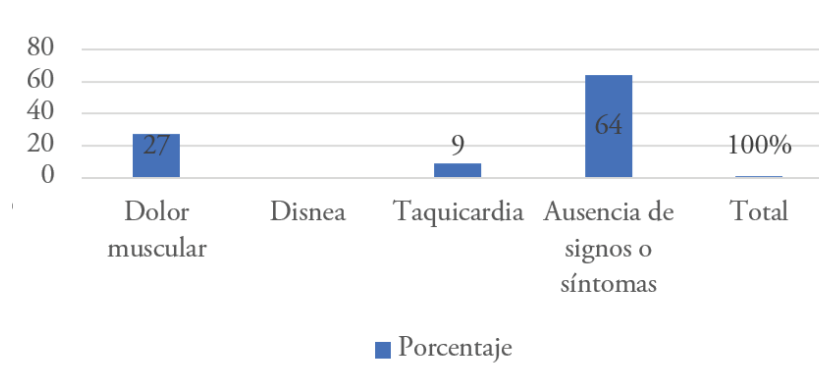

Figura 5. Signos y síntomas presentados como efectos secundarios a la realización del ejercicio en los pacientes de la unidad de hemodiálisis del Hospital Infantil Dr. Robert Reíd Cabral en el periodo abriljunio 2019

Fuente: elaboración propia.

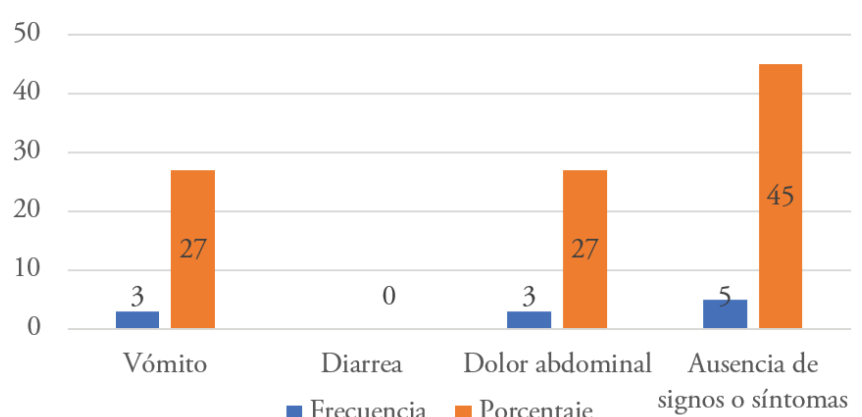

Figura 6. Signos y síntomas presentados como efecto secundario a la ingesta del suplemento en los pacientes de la unidad de hemodiálisis del Hospital Infantil Dr. Robert Reid Cabral en el periodo abriljunio 2019

Fuente: elaboración propia

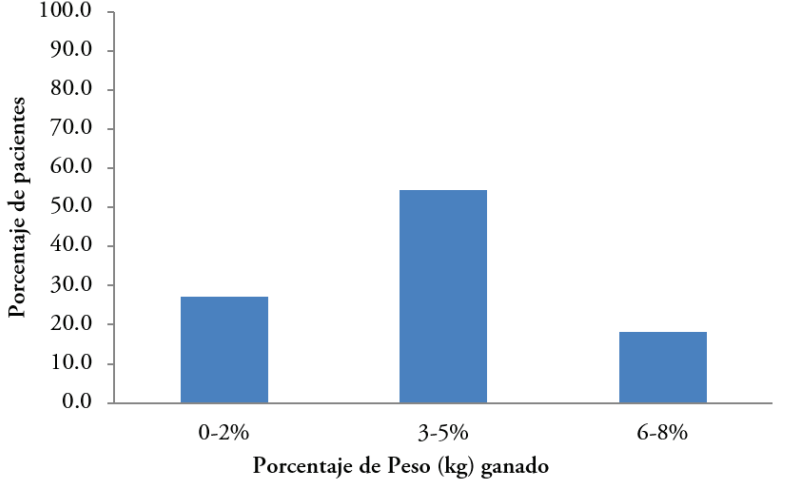

Figura 7. Evolución del peso tras la administración del suplemento y los ejercicios en los pacientes de la unidad de hemodiálisis del Hospital Infantil Dr. Robert Reid Cabral en el periodo abril-junio 2019

Fuente: elaboración propia.

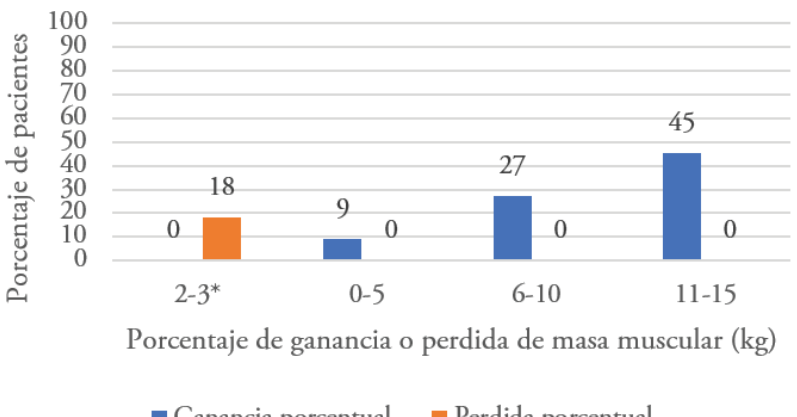

Figura 8. Evolución de la masa muscular en los pacientes de la unidad de hemodiálisis del Hospital Infantil Dr. Robert Reid Cabral en el periodo abriljunio 2019

Fuente: elaboración propia.

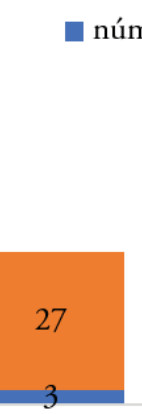

disminuý igual

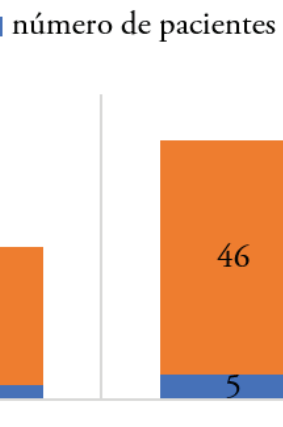

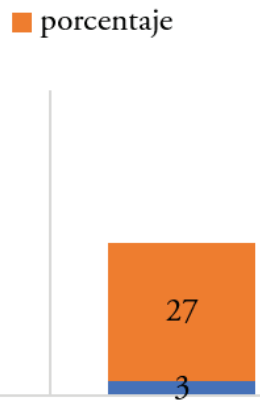

Figura 9. Evolución de los niveles de proteínas totales sérica de los pacientes en la unidad de hemodiálisis del Hospital Infantil Dr. Robert Reid Cabral en el periodo abril-junio 2019

Fuente: elaboración propia.

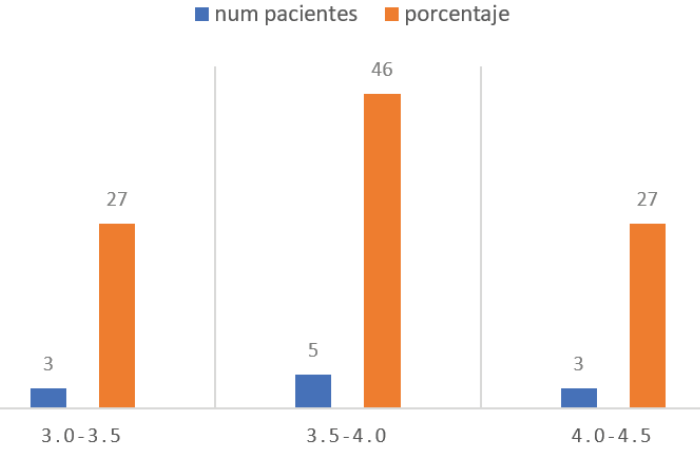

Figura 10. Evolución de los niveles de albumina sérica de los pacientes en la unidad de hemodiálisis del Hospital Infantil Dr. Robert Reid Cabral en el periodo abril-junio 2019

Fuente: elaboración propia.

Tabla 1. Cuadro estadístico de pruebas ${ }^{2}$

\begin{tabular}{|l|l|l|l|l|l|l|l|l|l|l|}
\hline & Peso & IMC & $\begin{array}{l}\text { Masa } \\
\text { grasa }\end{array}$ & $\begin{array}{l}\text { Masa } \\
\text { muscular }\end{array}$ & & $\begin{array}{l}\text { Niveles de } \\
\text { hemoglobina }\end{array}$ & $\begin{array}{l}\text { Niveles de } \\
\text { hematocrito }\end{array}$ & $\begin{array}{l}\text { Niveles } \\
\text { de urea }\end{array}$ & $\begin{array}{l}\text { Niveles de } \\
\text { proténa } \\
\text { total }\end{array}$ & $\begin{array}{l}\text { Niveles de } \\
\text { albumina }\end{array}$ \\
\hline $\mathbf{Z}$ & $-2,941^{\mathrm{b}}$ & $-2,699^{\mathrm{b}}$ &,$- 533^{\mathrm{c}}$ & & $-2,861^{\mathrm{b}}$ & $-2,224^{\mathrm{b}}$ & $-2,268^{\mathrm{b}}$ &,$- 934^{\mathrm{c}}$ &,$- 141^{\mathrm{c}}$ & $-1,378^{\mathrm{c}}$ \\
\hline $\mathbf{P}$ & .003 & .007 & .594 & & .004 & .026 & .023 & .350 & .888 & .168 \\
\hline
\end{tabular}

P-valor $=0.05$, esto implica un nivel de confianza del $95 \%$.

a. Prueba de Wilcoxon de los rangos con signo

b. Se basa en rangos negativos.

c. Se basa en rangos positivos.

Fuente: elaboración propia. 


\section{Discusión}

Es conocido que la presencia de malnutrición en los pacientes de diálisis implica un aumento de la morbimortalidad global por el incremento de la tasa de infecciones, hospitalización y estancia media. Se ha demostrado que el estado nutricional adecuado se asocia a mejor tolerancia de la enfermedad, respuesta favorable a la diálisis, aumento de la defensa contra las infecciones, mayor calidad de vida y bienestar.

Los estudios recientes indican que la suplementación de nutrientes por vía oral durante el proceso de diálisis podrían compensar la ingesta relativamente inadecuada de proteínas y de energía. En los últimos años se promueve el empleo de la nutrición enteral, por ser más fisiológica, económica y porque dispone de fórmulas de mayor calidad, que pueden ajustarse a las necesidades de los pacientes. Además, como consecuencia de la enfermedad renal crónica se ven afectados el sistema cardiovascular y músculo esquelético, que consecuentemente disminuyen la capacidad funcional del paciente.

En este estudio se describe por primera vez un programa para niños de la unidad de hemodiálisis del Hospital Infantil Doctor Robert Reid Cabral, donde se suplementa el paciente con los requerimientos energéticos y proteicos adecuados, además de incentivarlos para la realización de actividad física. En este caso, durante sus sesiones de hemodiálisis, practicamos ejercicios de resistencias ligeros y óptimos.

En el estudio de Pineda et al., 5 se describe que la población más frecuente de sus pacientes en hemopoblación más frecuente de sus pacientes en hemocomprendida en edades entre 10-18 años, coincidiendo esto con nuestra investigación, cuya población era igual de adolescentes con una edad promedio entre 13-18 años.

Asimismo, describen que un $91.6 \%$ de sus pacientes tuvo buena aceptabilidad del suplemento y fue consumido del 90-100 \% del producto, al igual que nuestros pacientes, $90 \%$ de estos tuvieron buena aceptabilidad.

Nuestro estudio difiere en que el sexo más frecuente de nuestra población fue masculino, para un $73 \%$ de la población estudiada, y en su estudio el sexo femenino.

El promedio de ejercicios de resistencia realizado por los pacientes fue de 20-30 minutos por sesiones, demostrando que el $54.5 \%$ de ellos realizaron sus sesiones de ejercicios correspondiente en el tiempo adecuado. En el año 2009, en Texas, ${ }^{6}$ se realizó un estudio piloto de actividad física intradiálisis para niños, donde pudieron identificar mejoras en la capacidad de ejercicio luego de tres meses. Los ejercicios se desarrollaron entre 30 y 60 minutos, dos veces por semana.

A principios de 1980, países como Estados Unidos comenzaron a implementar programas de ejercicio físico durante la hemodiálisis. Desde entonces y hasta hoy, los estudios refieren beneficios del ejercicio en este tipo de pacientes tanto a nivel fisiológico, como funcional o psicológico

Tras 30 años de investigación sobre los efectos del ejercicio de larga duración en pacientes en hemodiálisis se recomienda ser seguro y beneficioso. Pocos países de Latinoamérica tienen este programa como parte del tratamiento de los pacientes pediátricos en hemodiálisis, por primera vez es implementado en niños en nuestro país.

El signo más frecuente reportado en nuestro estudio como efecto secundario a la realización de ejercicios fue dolor muscular, para un $27 \%$. En el estudio de actividad física intradialisis ${ }^{6}$ a diferencia de nuestro estudio, los autores encontraron que la actividad física fue bien aceptada, no se produjeron efectos adversos en ninguno de sus pacientes.
Con relación a la sintomatología como efecto secundario de la ingesta del suplemento, se presentaron vómito y dolor abdominal en 3 pacientes, para un $27 \%$ durante el periodo del estudio. A diferencia del estudio publicado en la Revista Cubana pediátrica ${ }^{5}$, se observó la tolerancia del $100 \%$ de los pacientes por la ausencia de alteraciones como vómitos, diarreas o náuseas.

En los últimos años, se ha promovido el empleo de la nutrición enteral por ser más fisiológica, económica y porque dispone de fórmulas de mayor calidad, que pueden ajustarse a las necesidades de los pacientes. En la ERC con alto riesgo nutricional, se propone el uso de fórmulas con elevado contenido calórico $(2 \mathrm{kcal} / \mathrm{ML})$ y proteico (entre 1,3 y $1,4 \mathrm{~g} / \mathrm{kg}$ ), como la utilizada en nuestro estudio, con el aporte de vitaminas, oligoelementos y limitadas cantidades de vitamina A, fósforo, sodio y potasio.

El peso incrementó en un rango promedio de 3-5\% en un $54.5 \%$ de los pacientes, equivalente al incremento de 1-2 kg durante los meses del estudio.

Para el índice de masa corporal el porcentaje de ganancia fue de 0-6\% correspondiendo al $36 \%$ de los pacientes, equivalente a un IMC $17-21 \mathrm{~kg} / \mathrm{m}^{2}$. Amador, Waterlow y Lottom ${ }^{7}$ quienes señalan que la intervención nutricional permite una ganancia de peso de 30 a $50 \mathrm{~g} / \mathrm{kg} / \mathrm{d}$ ía, es decir, de 900 a $1,500 \mathrm{~g}$ en 4 semanas. Coinciden nuestros resultados con los del estudio de Pineda ${ }^{5}$, donde el mayor porcentaje de los pacientes mostró ganancia de peso, e índice de masa corporal de forma significativa.

La evolución de la masa muscular tuvo un rango promedio de incremento de $11-15 \%$ correspondiendo al $45 \%$ de los pacientes, esto equivale a una ganancia de músculos de 1-3 kg, luego de la suplementación oral y de la realización de los ejercicios de resistencia.

La evolución de la masa grasa, en $36 \%$ de los pacientes, disminuyó entre 6-14\% de grasa. Pineda et al. ${ }^{5}$ obtuvieron como resultado en su estudio que el incremento del área muscular no fue significativo $(\mathrm{p}<0,259)$ con relación al tiempo de ejercicio realizado. No encontramos ningún otro estudio donde se midiera la masa muscular o masa grasa sin bioimpedancia, por lo que no fue efectivo comparar con nuestro estudio.

En la evolución del perfil hemático, tanto la hemoglobina como el hematocrito aumentaron en el $100 \%$ de los pacientes, el $36 \%$ aumentó de $1-2$ gr de hemoglobina y el $64 \% 1-5 \%$ de hematocrito. Ninguno de los estudios revisados tomó en cuenta ticos en la población estudiada. El ejercicio aerobio eleva la capacidad de trabajo sobre la base del incremento del consumo de oxígeno, no solo de forma integral, sino también a nivel celular. Bernard y Holloszy ${ }^{4}$ refieren el incremento del número de mitocondrias como efecto de la práctica sistemática de actividades físicas, que se traduce en mayores posibilidades para consumir oxígeno, y disminuir la anemia.

Para los niveles de urea, un $27 \%$ de los pacientes disminuyeron entre 10-15\%, encontrándose además que un $37 \%$ aumentó sus niveles de 2-8 \%. A diferencia de Alonso MR et al., ${ }^{8}$ quienes en su artículo Evaluación de un programa de actividad fisica intradialítica, describen que el ejercicio durante la diálisis ha demostrado que mejora la eficiencia dialítica, aumentando la depuración de solutos tales como la urea. Sus pacientes mostraron un mayor porcentaje de reducción de urea posdiálisis con el programa de ejercicio impuesto.

Los niveles de proteínas totales permanecieron en rangos normales durante todo el estudio en $46 \%$ de los pacientes, al igual que la albumina, donde los rangos que mantuvieron fue de 3.5-4.0 g/dl (el mayor porcentaje de los pacientes aumentó en comparación a los niveles previo al estudio). Kuhlmann et al. ${ }^{9}$ demostraron un aumento significativo de la albúmina sérica en pacientes en hemodiálisis 
malnutridos, randomizados al recibir suplementos nutricionales por vía oral. En el estudio de Sharma ${ }^{10}$ los pacientes en HD malnutridos se aleatorizaron y recibieron un suplemento nutricional que aportaba $500 \mathrm{kcal}$ y $15 \mathrm{~g}$ de proteína durante 30 días, se vio un aumento significativo de la albúmina.

Este estudio es de gran impacto social, ya que por primera vez se realiza a nivel pediátrico un proyecto donde se suplementa el paciente y se realiza un programa de ejercicios sustentados en sus necesidades para su enfermedad de base.

No pudimos realizar grandes comparaciones por la falta de estudios de este tipo a nivel mundial, por lo que hemos realizado un gran aporte para continuar motivando a realizar proyectos como este con resultados positivos para nuestros pacientes.

\section{Conclusiones}

- El promedio de suplemento tomado por los pacientes de hemodiálisis durante el estudio fue de 7 onzas para un coeficiente de variabilidad del $23.6 \%$, demostrando que en el $90 \%$ de los pacientes ingirieron la cantidad que le correspondía.

- El promedio de ejercicios de resistencia realizado por los pacientes por mes fue de 187 minutos, estando dentro de los parámetros internaciones adecuados para un individuo en terapia dialítica. En este estudio significa que el $64 \%$ de los pacientes realizaron sus sesiones de ejercicios correspondientes en el tiempo adecuado.

- La edad más frecuente de los pacientes estudiados fue 17 años para un $45.5 \%$.

- El sexo más frecuente de los pacientes estudiados fue el masculino con un $73 \%$, correspondiendo a 8 pacientes.

- La evolución antropométrica de los pacientes estudiados estuvo dada por el incremento del peso y el índice de masa corporal. Para el peso aumentaron en un promedio $52 \mathrm{~kg}$, con un coeficiente de variabilidad del $30.3 \%$; para el índice de masa corporal un $91 \%$, en 10 pacientes aumentó mensualmente.

- Los signos y síntomas más frecuente como efecto secundario a la realización de ejercicios fue dolor muscular en 3 pacientes, para un $27 \%$ y el $64 \%$ de los pacientes no presentó ninguna otra sintomatología durante el período del estudio.

- La sintomatología más frecuente como efecto secundario de la ingesta del suplemento fue vómito, presentándose en 3 pacientes, al igual que el dolor abdominal, para un $27 \%$ durante el periodo del estudio.

- La evolución de la masa grasa en los pacientes estudiados presentó un promedio de variación de 22 , con un coeficiente de variación del $35.8 \%$, evidenciándose la disminución a través de los meses luego de implementarse la suplementación y los ejercicios físicos de resistencia.

- La evolución de la masa muscular o magra presenta una variación en promedio de 20 previo al estudio y 22 al finalizar, con un coeficiente de variación del $33 \%$, evidenciándose que el $75 \%$ de los pacientes aumentaron considerablemente su masa muscular durante el estudio.

- El cruce de variables, determinando el incremento de la masa muscular según la variación del peso mensual, obtuvo como resultado directamente proporcional que, a mayor aumento de peso mayor masa muscular, con un $\mathrm{R}^{2}: 0.94$

- La disminución de la masa grasa según el tiempo de ejercicio realizado tuvo como resultado que al practicar de 30-60 minutos por semana (120-240 minutos al mes) disminuimos la grasa corporal y aumentamos la masa muscular. $\mathrm{R}^{2}: 0.15$
- Una mayor ingesta de suplemento incrementa la masa muscular. $\mathrm{R}^{2:} 0.66$

- Los niveles de proteínas totales tuvieron un promedio de $6.7 \mathrm{mg} / \mathrm{dl}$, demostrando que el $73 \%$ de los pacientes permanecieron con niveles óptimos durante todo el estudio.

- Los niveles de albumina demuestran que al finalizar el estudio el $82 \%$ de los pacientes estudiados mantuvieron niveles promedios de $3.8 \mathrm{~m} / \mathrm{dl}$, siendo estos óptimos.

\section{Referencias}

1. Galindo P, Pérez de la Cruz A, Cerezo S, Martínez T, López P, Asensio C. Malnutrition and mortality in hemodialyzed patients. Nutr Hosp, 2001;16:27-30.

2. Star R. Treatment of acute renal failure. Kidney Int, 1998;54:1817-3

3. Cano N, Fiaccadori E, Tesinsky P, Toigo G, Druml W; DGEM (German Society for Nutritional Medicine), Kuhlmann M, Mann H, Hörl WH; ESPEN (European Society for Parenteral and Enteral Nutrition). ESPEN Guidelines on Enteral Nutrition: Adult renal failure. Clin Nutr. 2006 Apr;25(2):295-310. Doi: 10.1016/j. clnu.2006.01.023. Epub 2006 May 12. PMID: 16697495 .

4. Holloszy JO, Coyle, EF. (1984). Adaptations of skeletal muscle to endurance exercise and their metabolic consequences. Journal of Applied Physiology: Respiratory, Environmental and Exercise Physiology, 56(4),831-8. Available from: https://doi.org/10.1152/jappl. 1984.56.4.831
5. Pineda PérezS, Morell Contreras M, Valdés Martín S, Florin YrabierJ, Herrera Argüelles X, Hernández M. Soporte nutricional enteral en pacientes pediátricos con insuficiencia renal crónica en hemodiálisis. Rev Cubana Pediatr [Internet]. 2006 [citado el 28 de enero de 2022];78(1):0-0. Disponible en:http://scielo.sld.cu/scielo.php?script=sci arttext\&pid=S0034-7531200600010000

6. Stuart L, Goldstein LR. (2009). Un estudio piloto de ejercicio dos veces por semana durante la hemodiálisis en niños. Nefrología pediátrica, 24(4), 833-9.

7. Waterlow JC, Tomkins AM, Granthan MC, Gregor SM, Ops/Oms Washintong D. (1996). Malnutrición proteico energética. OPS/OMS Washintong D. 44-48.

8. Alonso Melgar A, Muley Alonso R. Nefrología al día. Diálisis pediátrica [Internet]. 2015 [citado el 28 de enero de 2022]. Disponible en: https:// www.nefrologiaaldia.org/57. Barcelona

9. Kuhlmann MK, Schmidt F, Kohler H. (1997). Oral nutritional support in malnourished patients on HD: preliminary results of a randomised controlled study (abstract). J Am Soc Nephrol, 8.

10. Sharma M, Rao M, Jacob S, Jacob CK. (2002). A controlled trial of intermittent enteral nutrient supplementation in maintenance hemodialysis patients. Journal of Renal Nutrition: The Official Journal of the Council on Renal Nutrition of the National Kidney Foundation, 12(4),229-37. Available from: https://doi.org/10.1053/jren. 2002.35300 